\title{
Modeling of distributed piezoelectric actuators integrated with thin cylindrical shells
}

\author{
Su-Wei Zhou, Chen Liang, and Craig A. Rogers \\ Center for Intelligent Material Systems and Structures, Virginia Polytechnic Institute and State University, \\ Blacksburg, Virginia 24061-0261
}

(Received 5 August 1993; revised 21 March 1994; accepted 13 May 1994)

\begin{abstract}
The dynamic interaction between induced strain piezoelectric (PZT) actuators and their host structures is often ignored in the modeling of intelligent structures. A more realistic investigation of intelligent material systems must account for the dynamic behaviors of integrated actuator/substrate systems. In this paper, a generic method for the dynamic modeling of distributed PZT actuator-driven thin cylindrical shells has been developed using a mechanical impedance approach. The impedance characteristics of a cylinder corresponding to the excitation of a pair of pure bending moments have been developed, from which the dynamic output moments (or forces) of PZT actuators can be accurately predicted. Direct comparisons have been made between a conventional static modeling approach and the impedance method in order to identify the critical differences between these modeling methods for thin cylindrical structures. The case studies demonstrate that the mechanical impedance matching between PZT actuators and host structures has an impact on the output performance of the actuators. The dynamic essence of integrated PZT/substrate systems has thus been revealed.
\end{abstract}

PACS numbers: $43.40 . \mathrm{Vn}, 43.40 . \mathrm{Ey}, 43.38 . \mathrm{Fx}$

\section{INTRODUCTION}

The use of piezoelectric materials (PZT) as actuators and sensors in intelligent structures has drawn much attention in the active controls community. An accurate mathematical description of integrated PZT/substrate systems is indispensable in the development of practical applications of PZT materials in active vibration control and active structural acoustic control. One investigation devoted to the modeling of actively controlled cylinders was based on the layered shell theory (Tzou, 1989); in this study, a completely distinct layer of the piezoelectric material was used to make up a composite cylinder. A more convenient and feasible configuration of PZT actuators in adaptive structures is the use of segmented piezoelectric patches bonded on or embedded in host structures. For the modeling of these PZT actuators locally coupled with shell structures, static approaches have been used to estimate the induced loading. Sonti and Jones (1991) investigated the performance of the controlled cylinder using the piezoactuator control patch and a point force for both on- and off-resonance cases. The output performance of the PZT actuator, however, was not specifically studied. The assumption was made in the modeling that the dimension of the PZT patch in the circumferential direction is kept small relative to the cylinder radius. The curvature effects could then be neglected. The static models developed from thin plate theory (Crawley and Lazarus, 1991; Dimitriadis et al., 1989; Wang and Rogers, 1991) could then be used in smart shell structures. Lester and Lefebvre (1991) applied the same assumption in their study and researched both outof-plane and in-plane piezoelectric actuation for controlling sound radiation and transmission related to vibrating cylinders. It is typically assumed in the static models that the added mass and stiffness of PZT actuators are insignificant.
The dynamic interaction between the PZT actuator and the host structure is ignored. Thus, the input impedance of the actuators and the mechanical impedance of the host structures are not included in the modeling. The static analysis usually leads to the conclusion that the amplitude of the excitation force of the PZT actuator is independent of host structural dynamics and frequency.

A more realistic investigation must account for the dynamic properties of the actuators, the actuators have their own mechanical impedance. When an active force provided by the PZT actuator is applied to a host structure, the PZT itself is driven by the force. Therefore, the mechanical resonance of an integrated PZT/substrate system is the combination of the dynamic behavior of the PZT actuator and that of the actuated structure. Hagood et al. (1990) proposed a dynamic model based on Rayleigh-Ritz energy formulation. The parasite mass and stiffness of the integrated PZT patch was included in the governing equation of the system. The actively controlled cantilevered beam was tested and favorable results were obtained. As the active control force needs to be calculated, the blocking force was used. Liang et al. (1993) used impedance analysis in modeling of a PZT actuator-driven one-degree-of-freedom spring-mass-damping system. The frequency-dependent output performance of the PZT actuator was predicted. Rossi et al. (1993) then applied the impedance approach to model PZT actuator-driven circular rings and performed the experiments for validating the theoretical model. Nevertheless, the previous studies related to the dynamic modeling for shell structures have so far been limited to the one-dimensional systems. For general twodimensional shell structures, the mechanical impedance coupling in different coordinate directions occurs. An extensive investigation is thus highly required.

The current work is focused on analysis of two- 


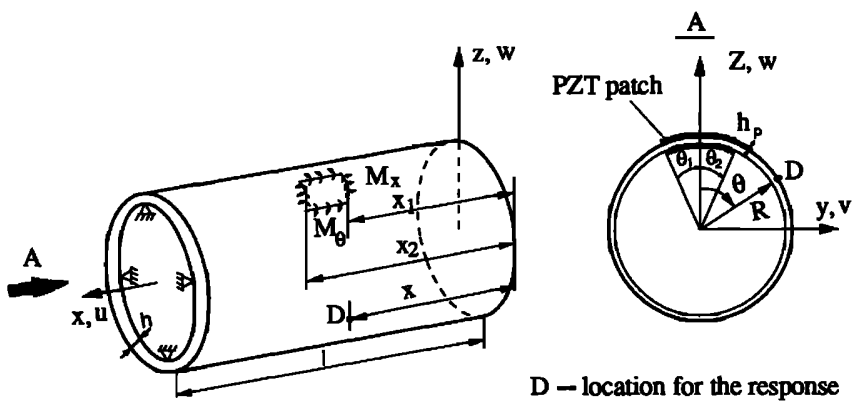

FIG. 1. A thin cylindrical shell with surface-bonded PZT actuators.

dimensional thin cylindrical shells excited by a pair of PZT actuators in a pure bending mode. A generic method for the impedance modeling of integrated PZT/shell systems will be presented. The numerical case studies and comparisons based upon a simply supported cylinder will be performed to demonstrate the significant difference between a conventional static modeling approach and the impedance method. The results will show that the excitation force of the induced strain actuators depends on the mechanical impedance matching between the PZT actuators and the cylinder. The physics of the dynamic interaction between induced strain actuators and host structures will be revealed.

\section{IMPEDANCE MODEL OF INTEGRATED PZT/SHELL SYSTEMS}

A physical model of a thin cylindrical shell excited by PZT actuators is shown in Fig. 1. The PZT patches are assumed to be perfectly bonded on the internal and external surfaces of the cylinder so that a pure bending moment excitation can be locally created. Figure 2 displays the corresponding impedance model. The dynamic behavior of the cylinder in the $x$ and $\theta$ direction is represented by the direct impedance $Z_{x x}$ and $Z_{\theta \theta}$ as well as the cross impedance $Z_{x \theta}$ and $Z_{\theta x}$, respectively. Under the actuation of the moments, $M_{x}$ and $M_{\theta}$, the angular velocity response of the cylinder at the edge of the bonded PZT patches may be described by

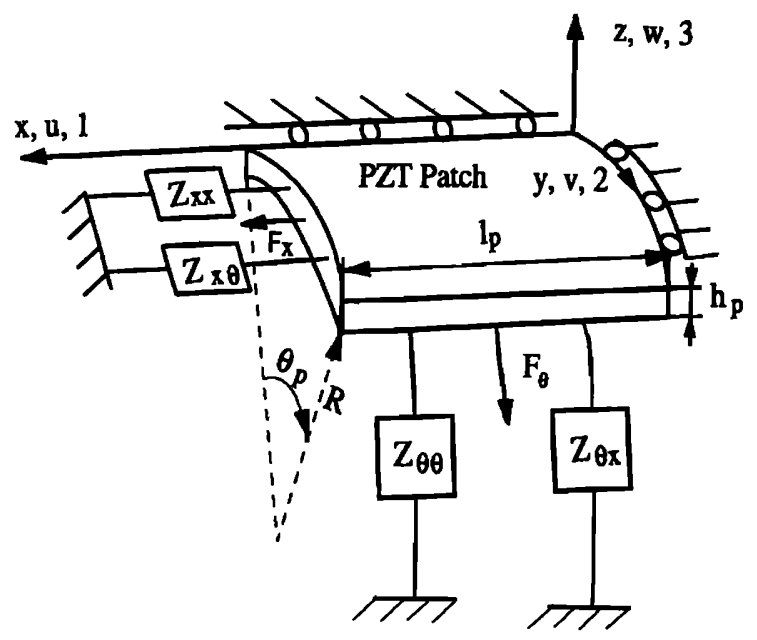

FIG. 2. A physical model of a PZT actuator integrated with a thin shell structure represented by mechanical impedance.

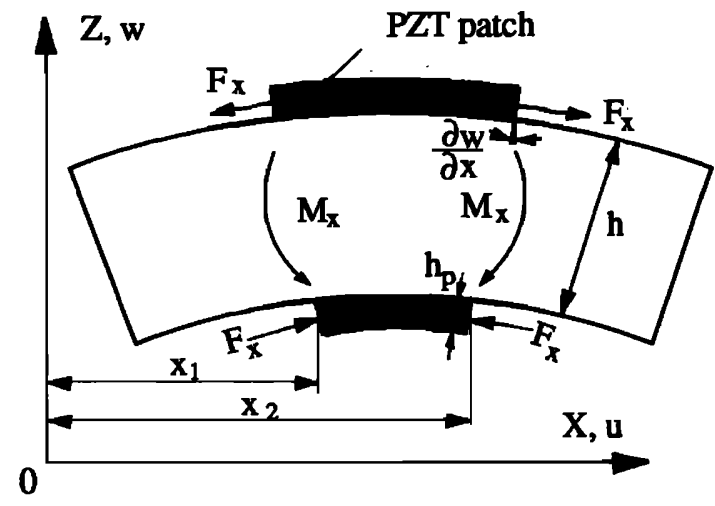

FIG. 3. The angular deformation of a cylinder in the $x$ direction, actuated by a pair of moments.

$$
\begin{aligned}
\nabla \beta_{x} & =\left(\frac{\partial \dot{w}}{\partial x}\right)_{x=x_{2}}-\left(\frac{\partial \dot{w}}{\partial x}\right)_{x=x_{1}}=-\left(H_{x x} M_{x}+H_{\theta x} M_{\theta}\right), \\
\nabla \beta_{\theta} & =\left(\frac{\partial \dot{w}}{R \partial \theta}\right)_{\theta=\theta_{2}}-\left(\frac{\partial \dot{w}}{R \partial \theta}\right)_{\theta=\theta_{1}} \\
& =-\left(H_{x \theta} M_{x}+H_{\theta \theta} M_{\theta}\right),
\end{aligned}
$$

where $\nabla \beta$ is the total angular deformation of the cylinder; $R$ is the radius of the cylinder. The minus sign indicates that the structural reactions are equal and opposite to the output forces of the PZT actuator. Here, $H_{x x}$ and $H_{\theta \theta}$ are the direct admittance, $H_{x \theta}$ and $H_{\theta x}$ are the cross admittance of the cylinder and responsible for the coupling of the input moments in the $x$ and $\theta$ directions. The moment admittance is defined as

$$
H_{l k}=\frac{\nabla \beta_{k}}{M_{l}} \quad(l, k=x, y) .
$$

Figure 3 shows the geometric deformation of the cylinder in the $x$ direction under the actuation of $M_{x}$. The similar deformation occurs in the $\theta$ direction. Considering the relationship between the translational displacement and the angular deformation of the cylinder, the in-plane displacement, $u$ and $v$, can be expressed by

$$
\begin{aligned}
& u=\frac{h+h_{p}}{2}\left[\left(\frac{\partial w}{\partial x}\right)_{x=x_{2}}-\left(\frac{\partial w}{\partial x}\right)_{x=x_{1}}\right], \\
& v=\frac{h+h_{p}}{2}\left[\left(\frac{\partial w}{R \partial \theta}\right)_{\theta=\theta_{2}}-\left(\frac{\partial w}{R \partial \theta}\right)_{\theta=\theta_{1}}\right],
\end{aligned}
$$

where $h$ and $h_{p}$ are the thicknesses of the cylinder and the PZT actuator, respectively. The corresponding in-plane velocity, $\dot{u}$ and $\dot{v}$, are then given by

$$
\begin{aligned}
& \dot{u}=\frac{h+h_{p}}{2}\left[\left(\frac{\partial \dot{w}}{\partial x}\right)_{x=x_{2}}-\left(\frac{\partial \dot{w}}{\partial x}\right)_{x=x_{1}}\right], \\
& \dot{v}=\frac{h+h_{p}}{2}\left[\left(\frac{\partial \dot{w}}{R \partial \theta}\right)_{\theta=\theta_{2}}-\left(\frac{\partial \dot{w}}{R \partial \theta}\right)_{\theta=\theta_{1}}\right] .
\end{aligned}
$$


Using the relation of the in-plane force and the out-of-plane moment: $M_{x(\theta)}=\left(h+h_{p}\right) F_{x(\theta)}$ and substituting Eq. (4) into Eq. (1) results in

$$
\begin{aligned}
\left(\begin{array}{c}
F_{x} \\
F_{\theta}
\end{array}\right) & =-\frac{2}{\left(h+h_{p}\right)^{2}}\left(\begin{array}{ll}
H_{x x} & H_{\theta x} \\
H_{x \theta} & H_{\theta \theta}
\end{array}\right)^{-1}\left(\begin{array}{c}
\dot{u} \\
\dot{v}
\end{array}\right) \\
& =-\left(\begin{array}{ll}
Z_{x x} & Z_{x \theta} \\
Z_{\theta x} & Z_{\theta \theta}
\end{array}\right)\left(\begin{array}{l}
\dot{u} \\
\dot{v}
\end{array}\right),
\end{aligned}
$$

where the structural impedance $Z_{l k}(l, k=x, y)$ can be derived from classic shell theory and will be developed later; $\dot{u}$ and $\dot{v}$ need to be solved so that the output force can be quantitatively predicted.

When an external electric field is applied to PZT patches along the polarization direction (3), the equation of motion of the PZT actuator shown in Fig. 2 may be expressed by

$$
\begin{aligned}
& \rho_{p} \frac{\partial^{2} u}{\partial t^{2}}=Y_{p 11}^{E} \frac{\partial^{2} u}{\partial x^{2}}, \\
& \rho_{p} \frac{\partial^{2} v}{\partial t^{2}}=Y_{p 22}^{E} \frac{\partial^{2} v}{R \partial \theta^{2}},
\end{aligned}
$$

where the subscript $p$ refers to the parameters of the PZT actuator, and 11 and 22 denote the coordinates of PZT materials; $\rho$ is the mass density and $Y^{E}$ is the Young's modulus. The solution of Eq. (6) is described by

$$
\begin{aligned}
& u=\left[A \sin \left(k_{p 11} x\right)+B \cos \left(k_{p 11} x\right)\right] e^{j \omega t}, \\
& v=\left[C \sin \left(k_{p 22} R \theta\right)+D \cos \left(k_{p 22} R \theta\right)\right] e^{j \omega t},
\end{aligned}
$$

where $A, B, C$, and $D$ are unknowns and can be determined by the boundary conditions; $j$ symbolizes the imaginary part of a complex number; $\omega$ is the input angular frequency. Considering the isotropy of the PZT material in the 1 and 2 directions yields the wave number:

$$
k_{p}^{2}=k_{p 11}^{2}=k_{p 22}^{2}=\omega^{2}\left(\rho_{p} / Y_{p}^{E}\right) .
$$

Applying the displacement boundary conditions, $u_{x=0}=0$ and $v_{\theta=0}=0$ to Eqs. (7), leads to $B=D=0$. The unknowns, $A$ and $C$, can be determined from the constitutive equation of the PZT actuator at $x=l_{p}$ and $\theta=\theta_{p}$ :

$$
\begin{aligned}
\left(\begin{array}{c}
\varepsilon_{x} \\
\varepsilon_{y}
\end{array}\right)=\left(\begin{array}{c}
\frac{\partial u}{\partial x} \\
\frac{\partial v}{R \partial \theta}
\end{array}\right)= & \left(\begin{array}{cc}
\frac{1}{Y_{p}^{E} R \theta_{p} h_{p}} & -\frac{\nu_{p}}{Y_{p}^{E} l_{p} h_{p}} \\
-\frac{\nu_{p}}{Y_{p}^{E} R \theta_{p} h_{p}} & \frac{1}{Y_{p}^{E} l_{p} h_{p}}
\end{array}\right)\left(\begin{array}{l}
F_{x} \\
F_{\theta}
\end{array}\right) \\
& +\left(\begin{array}{l}
d_{31} \\
d_{32}
\end{array}\right) E,
\end{aligned}
$$

where $l_{p}, \theta_{p}$, and $d_{31(2)}$ are the length, the angle, and the piezoelectric constant of the PZT actuator, respectively. Note that the Poisson's ratio of the PZT material, $\nu_{p}$ is introduced so that the mechanical coupling of the in-plane motion in different directions $(x, \theta)$ of the PZT actuator can be included in the modeling. Substituting Eqs. (5) and (7) into Eq. (9) and taking the algebraic operation to rearrange $A$ and $C$ yield:

$$
\begin{aligned}
& \left(\begin{array}{cc}
C_{l}\left(1-\frac{\nu_{p}}{\alpha} \frac{Z_{x \theta}}{Z_{p x x}}+\frac{Z_{x x}}{Z_{p x x}}\right) & C_{\theta}\left(\alpha \frac{Z_{\theta x}}{Z_{p \theta \theta}}-\nu_{p} \frac{Z_{\theta \theta}}{Z_{p \theta \theta}}\right) \\
C_{l}\left(\frac{1}{\alpha} \frac{Z_{x \theta}}{Z_{p x x}}-\nu_{p} \frac{Z_{x x}}{Z_{p x x}}\right) & C_{\theta}\left(1-\nu_{p} \alpha \frac{Z_{\theta x}}{Z_{p \theta \theta}}+\frac{Z_{\theta \theta}}{Z_{p \theta \theta}}\right)
\end{array}\right) \\
& \times\left(\begin{array}{l}
A \\
C
\end{array}\right)=\left(\begin{array}{l}
d_{31} \\
d_{32}
\end{array}\right) E,
\end{aligned}
$$

where $\quad C_{l}=k_{p} \cos \left(k_{p} l_{p}\right) \quad$ and $\quad C_{\theta}=k_{p} \cos \left(k_{p} R \theta_{p}\right)$; $\alpha=l_{p} /\left(R \theta_{p}\right)$ is the ratio of the length to the width of the PZT patch; $Z_{p x x}$ and $Z_{p \theta \theta}$ are the input impedances of the PZT actuator in the $x$ and $\theta$ directions, defined as

$$
\begin{aligned}
& Z_{p x x}=K_{p x} \frac{k_{p} l_{p}}{\tan \left(k_{p} l_{p}\right)} \frac{1}{j \omega}, \\
& Z_{p \theta \theta}=K_{p \theta} \frac{k_{p} R \theta_{p}}{\tan \left(k_{p} R \theta_{p}\right)} \frac{1}{j \omega},
\end{aligned}
$$

with the static extension stiffnesses of the actuator in the $x$ and $\theta$ directions, $K_{p x}=Y^{E}{ }_{p} R \theta_{p} h_{p} / l_{p} \quad$ and $K_{p \theta}=Y^{E}{ }_{p} l_{p} h_{p} /\left(R \theta_{p}\right)$. Solving for $A$ and $C$ from Eq. (10) and substituting it into Eqs. (7), the displacement and velocity responses of the PZT actuator are obtained. The dynamic force output of the PZT actuator can then be determined from Eq. (5):

$$
\begin{aligned}
& F_{x}=\bar{F}_{x} e^{j \omega t}=-j \omega\left(A S_{l} Z_{x x}+C S_{\theta} Z_{x \theta}\right) e^{j \omega t}, \\
& F_{\theta}=\bar{F}_{\theta} e^{j \omega t}=-j \omega\left(A S_{l} Z_{\theta x}+C S_{\theta} Z_{\theta \theta}\right) e^{j \omega t},
\end{aligned}
$$

where $S_{l}=\sin \left(k_{p} l_{p}\right)$ and $S_{\theta}=\sin \left(k_{p} R \theta_{p}\right)$. Accordingly, the amplitude of the line moments per unit length created by the pair of PZT patches, $\bar{M}_{x,(\theta)}$ (in N m/m), can be determined as

$$
\begin{gathered}
\bar{M}_{x}=\frac{\bar{F}_{x}\left(h+h_{p}\right)}{R \theta_{p}}=-j \frac{\omega\left(h+h_{p}\right)\left(A S_{l} Z_{x x}+C S_{\theta} Z_{x \theta}\right)}{R \theta_{p}}, \\
\bar{M}_{\theta}=\frac{\bar{F}_{\theta}\left(h+h_{p}\right)}{l_{p}}=-j \frac{\omega\left(h+h_{p}\right)\left(A S_{l} Z_{\theta x}+C S_{\theta} Z_{\theta \theta}\right)}{l_{p}} .
\end{gathered}
$$

The distributed line moments are thus expressed by

$$
\begin{aligned}
M_{x}= & \bar{M}_{x}\left[\delta\left(x-x_{1}\right)-\delta\left(x-x_{2}\right)\right]\left[h\left(\theta-\theta_{1}\right)\right. \\
& \left.-h\left(\theta-\theta_{2}\right)\right] e^{j \omega t}, \\
M_{\theta}= & \bar{M}_{\theta}\left[\delta\left(\theta-\theta_{1}\right)-\delta\left(\theta-\theta_{2}\right)\right]\left[h\left(x-x_{1}\right)\right. \\
& \left.-h\left(x-x_{2}\right)\right] e^{j \omega t},
\end{aligned}
$$

where $\delta(x)$ and $\delta(\theta)$ are the Dirac delta functions; $h(x)$ and $h(\theta)$ are the Heaviside functions; $x_{1}, x_{2}, \theta_{1}$, and $\theta_{2}$ are the location coordinate of the edge of PZT patches on the cylinder, as illustrated in Fig. 1.

Since the coefficients $A$ and $C$, as well as the mechanical impedance of the cylinder in Eqs. (13), are functions of the frequency, the moment outputs of the PZT actuator are frequency dependent. If the cross impedance $Z_{x \theta}$ and $Z_{\theta x}$ in Eq. (10) is assumed to be zero and the Poisson's effect of the PZT material is ignored, a decoupled analysis is then ob- 
tained for one-dimensional rings. The formulation for the force output of the PZT actuator expressed in Eq. (12) is reduced to

$$
F_{\theta}=-\frac{Z}{Z+Z_{p}} Y_{p}^{E} S_{p} d_{32} E,
$$

where $Z$ is the mechanical impedance of the ring, $Z_{p}$ is the input impedance of the PZT actuator, and $S_{p}$ is the cross section area of the PZT actuator. Equation (15) is same as the formulation that was derived from the circular rings by Rossi et al. (1993). The two-dimensional impedance analysis, therefore, is applicable to one-dimensional structures.

Thus far, the dynamic forces and moments of the PZT actuator have been obtained based upon the impedance characteristics of the PZT and the cylinder. The input impedance of the PZT actuator is given by Eq. (11). The mechanical impedance of the cylinder is determined by the inverse admittance matrix in Eq. (5), which depends on the location of the actuators, the structural configuration, the boundary condition, and the physical properties. The next section will address the calculation of the admittance of the cylinder actuated by line moments.

\section{ADMITTANCE CALCULATION OF A CYLINDER}

The general solution of the response of a thin shell excited by line moments can be determined by solving the following Love equations (Soedel, 1981):

$$
\begin{aligned}
& L_{u}(u, v, w)-\lambda \dot{u}-\rho h \ddot{u}=-q_{u}-\frac{1}{2 R} \frac{\partial M_{n}}{\partial \theta}, \\
& L_{v}(u, v, w)-\lambda \dot{v}-\rho h \ddot{v}=-q_{v}-\frac{1}{2} \frac{\partial M_{n}}{\partial x}, \\
& L_{w}(u, v, w)-\lambda \dot{w}-\rho h \ddot{w}=-q_{w}-\frac{1}{R}\left(R \frac{\partial M_{x}}{\partial x}+\frac{\partial M_{\theta}}{R \partial \theta}\right),
\end{aligned}
$$

where $q_{i}(i=u, v, w)$ is the pressure applied along the $x, y$, and $z$ directions normal to the surface of the shell, respectively. The twisting moment $M_{n}$ is applied about the normal (z) direction. $\lambda$ is an equivalent viscous damping factor. The operator $L_{i}(i=u, v, w)$ can be evaluated from an eigenvalue analysis. Since the transverse modes in a very shallow shell are the dominant modes, it is assumed that the inertial effects in the in-plane directions are neglected and the loading is applied normally to the surface of the shells (Soedel, 1981; Leissa, 1973). In the current case, the ratio of the thickness to the radius of the cylinder is assumed to be $1 / 100$ and the theory for thin shells is thus applied. Only Eq. (16c) is considered in the modeling. The modal expansion series solution of Eq. (16c) is expressed by

$$
w=\sum_{m=1}^{\infty} \sum_{n=0}^{\infty} p_{m n} W_{m n}(x, \theta),
$$

where $m$ and $n$ refer to the axial and circumferential mode numbers; $p_{m n}$ is the modal participation factor; $W_{m n}(x, \theta)$ is the eigenfunction. The operator $L_{w}$ is evaluated from the eigenvalue analysis:
TABLE I. Material properties of the PZT (Piezo Systems, Inc., 1987) and aluminum.

\begin{tabular}{lccccc}
\hline \hline & $\begin{array}{c}Y^{E}\left(\mathrm{~N} / \mathrm{m}^{2}\right) \\
\left(\times 10^{10}\right)\end{array}$ & $\begin{array}{c}\rho \\
\left(\mathrm{kg} / \mathrm{m}^{3}\right)\end{array}$ & $\nu$ & $\begin{array}{c}d_{31}, d_{32}(\mathrm{~m} / \mathrm{V}) \\
\left(\times 10^{-10}\right)\end{array}$ & $\xi_{m n}$ \\
\hline PZT & 6.3 & 7650 & 0.3 & -1.66 & 0.005 \\
Aluminum & 6.9 & 2700 & 0.33 & $\mathrm{~N} / \mathrm{A}$ & 0.005 \\
\hline \hline
\end{tabular}

$$
L_{w}\left(w_{m n}\right)=-\rho h \omega_{m n}^{2} w_{m n},
$$

where $\omega_{m n}$ is the natural frequency of the cylinder. Substituting Eqs. (17) and (18) into Eq. (16c) and assuming $q_{w}=0$ results in

$$
\begin{aligned}
& \sum_{m=1}^{\infty} \sum_{n=0}^{\infty}\left(\rho h \ddot{p}_{m n}+\lambda \dot{p}_{m n}+\rho h \omega_{m n}^{2} p_{m n}\right) W_{m n}(x, \theta) \\
& \quad=\frac{1}{R}\left(R \frac{\partial M_{x}}{\partial x}+\frac{\partial M_{\theta}}{R \partial \theta}\right) .
\end{aligned}
$$

For a simply supported cylinder, the eigenfunction $W_{m n}(x, \theta)$ can be described by

$$
W(x, \theta)=\sin (m \pi / l) x \cos n(\theta-\psi),
$$

where $\psi$ is the relative angular location of the input moment with respect to the coordinate system and $l$ is the length of the cylinder. Substituting Eq. (20) into Eq. (19) and using the usual modal expansion technique yields:

$$
\ddot{p}_{m n}+2 \xi_{m n} \dot{p}_{m n}+\omega_{m n}^{2} p_{m n}=F_{m n} e^{j \omega t},
$$

where the equivalent forcing function, $F_{m n}$, is of the form

$$
\begin{aligned}
F_{m n}= & \frac{1}{\rho h N_{m n}} \int_{0}^{l} \int_{0}^{2 \pi} W_{m n}(x, \theta) \\
& \times\left(R \frac{\partial M_{x}}{\partial x}+\frac{\partial M_{\theta}}{R \partial \theta}\right) d x d \theta,
\end{aligned}
$$

where

$$
\begin{aligned}
N_{m n} & =\int_{0}^{l} \int_{0}^{2 \pi} \sin ^{2} \frac{m \pi x}{l} \cos ^{2} n(\theta-\psi) R d x d \theta \\
& = \begin{cases}R l \pi / 2 & (n \neq 0), \\
R l \pi & (n=0) .\end{cases}
\end{aligned}
$$

In the case of $\psi=0$, Eq. (22) becomes

$$
\begin{aligned}
F_{m n 1}= & \frac{C_{x}}{\rho h N_{m n}}\left(\frac{R \pi m}{l n} \bar{M}_{x}+\frac{\ln }{R \pi m} \bar{M}_{\theta}\right) \\
& \times\left(\sin n \theta_{1}-\sin n \theta_{2}\right),
\end{aligned}
$$

where $C_{x}=\cos \left(m \pi x_{1} / l\right)-\cos \left(m \pi x_{2} / l\right)$. In the case of $\psi=\pi / 2 n$,

$$
\begin{aligned}
F_{m n 2}= & \frac{C_{x}}{\rho h N_{m n}}\left(\frac{R m \pi}{\ln } \bar{M}_{x}+\frac{\ln }{R m \pi} \bar{M}_{\theta}\right) \\
& \times\left(\cos n \theta_{2}-\cos n \theta_{1}\right) .
\end{aligned}
$$

In particular, when $\psi=0$ and $n=0$, the effective force function is reduced to 


$$
F_{m 0}=\frac{C_{x}}{\rho h} \frac{m}{l^{2}}\left(\theta_{1}-\theta_{2}\right) \bar{M}_{x} .
$$

The solution of the governing equation (21) is expressed by

$$
p_{m n}(t)=\frac{F_{m n} e^{j\left(\omega t-\phi_{m n}\right)}}{\omega_{m n}^{2} \sqrt{\left(1-\left(\omega / \omega_{m n}\right)^{2}\right)^{2}+4 \xi_{m n}^{2}\left(\omega / \omega_{m n}\right)^{2}}}
$$

with the phase shift,

$$
\phi_{m n}=\tan ^{-1} \frac{2 \xi_{m n}\left(\omega / \omega_{m n}\right)}{1-\left(\omega / \omega_{m n}\right)^{2}},
$$

where $\xi_{m n}$ is the modal damping coefficient. The transverse displacement response of the cylinder is thus obtained:

$$
w(x, \theta, t)=\sum_{m=1}^{\infty} \sum_{n=0}^{\infty} \frac{\left(F_{m n 1} \cos n \theta+F_{m n 2} \sin n \theta\right) \sin (m \pi / l) x e^{j\left(\omega t-\phi_{m n}\right)}}{\omega_{m n}^{2} \sqrt{\left[1-\left(\omega / \omega_{m n}\right)^{2}\right]^{2}+4 \xi_{m n}^{2}\left(\omega / \omega_{m n}\right)^{2}}}
$$

Substituting Eq. (29) into Eq. (1) and recalling the definition of the admittance in Eq. (2), the direct admittance at the middle point of the edge of the PZT actuator can be determined as

$$
\begin{aligned}
H_{x x}= & \frac{4 \omega \pi}{\rho h R \theta_{p} l^{3}} \sum_{m=1}^{\infty} \sum_{n=1}^{\infty}\left(\frac{m^{2} C_{x}^{2} \sin \left[n\left(\theta_{2}-\theta_{1}\right) / 2\right]}{n \Delta_{m n}}\right) \\
& \times e^{j\left(\pi / 2-\phi_{m n}\right)}
\end{aligned}
$$

and

$$
\begin{aligned}
H_{\theta \theta}= & \frac{4 \omega}{\rho h l_{p} \pi^{2} R^{3}} \sum_{m=1}^{\infty} \sum_{n=1}^{\infty}\left(\frac{n^{2} C_{x} S_{x}\left[1-\cos n\left(\theta_{1}-\theta_{2}\right)\right]}{m \Delta_{m n}}\right) \\
& \times e^{j\left(\pi / 2-\phi_{m n}\right)}
\end{aligned}
$$

where $S_{x}=\sin \left[m \pi\left(x_{1}+x_{2}\right) /(2 l)\right]$ and $\Delta_{m n}$ is given by

$$
\Delta_{m n}=\omega_{m n}^{2} \sqrt{\left(1-\left(\omega / \omega_{m n}\right)^{2}\right)^{2}+4 \xi_{m n}^{2}\left(\omega / \omega_{m n}\right)^{2}} .
$$

Similarly, the cross admittances are obtained by

$$
\begin{aligned}
H_{x \theta}= & \frac{4 \omega}{\rho h \theta_{p} R^{2} l^{2}} \sum_{m=1}^{\infty} \sum_{n=1}^{\infty}\left(\frac{m C_{x} S_{x}\left[1-\cos n\left(\theta_{1}-\theta_{2}\right)\right]}{\Delta_{m n}}\right) \\
& \times e^{j\left(\pi / 2-\phi_{m n}\right)}
\end{aligned}
$$

and

$$
\begin{aligned}
H_{\theta x}= & \frac{4 \omega}{\rho h \pi R^{2} l l_{p}} \sum_{m=1}^{\infty} \sum_{n=1}^{\infty}\left(\frac{n C_{x}^{2} \sin \left[n\left(\theta_{2}-\theta_{1}\right) / 2\right]}{\Delta_{m n}}\right) \\
& \times e^{j\left(\pi / 2-\phi_{m n}\right)} .
\end{aligned}
$$

TABLE II. The geometric size of the PZT actuator and the cylinder (unit: $\mathrm{mm})$.

\begin{tabular}{lcccc}
\hline \hline & Length & Radius & Angle & \\
& $l$ & $R$ & $\theta_{p}$ (degree $)$ & Thickness \\
\hline PZT & 80 & 200 & 6 & $0.2,0.5,1.0$ \\
Cylinder & 700 & 200 & 360 & 2.0 \\
\hline \hline
\end{tabular}

\section{NUMERICAL EXAMPLES AND DISCUSSION}

The simply supported thin cylinder used in the case study is made of aluminum and its geometric configuration is shown in Fig. 1. The material properties of aluminum and PZT (G1195) are listed in Table I and their geometric parameters are given in Table II. In the case study, two geometric parameters, the thickness of the PZT $\left(h_{p}=0.2,0.5,1.0 \mathrm{~mm}\right)$ and the location of the PZT on the cylinder $\left(x_{1}=310,100\right.$ $\mathrm{mm}$ ) are examined. The purpose is to identify the effect of the input impedance of the PZT and the structural impedance on the moment outputs of the PZT actuator and to determine how they influence the response of the cylinder.

For comparison of modeling approaches, the static line moment calculation is based upon a flat PZT patch, because the dimension of the PZT patch in circumferential direction is kept small relative to the cylinder radius, thus the curvature effects can be neglected (Sonti and Jones, 1991; Lester and Lefebvre, 1991). This is a reasonable approximation from the experimental point of view. In the current numerical examples, the circumferential size of the PZT patch is much smaller than that of the cylinder, $\left(R \theta_{p}\right) / R=1 / 10$, hence flat PZT patches are used in the static calculations. There are

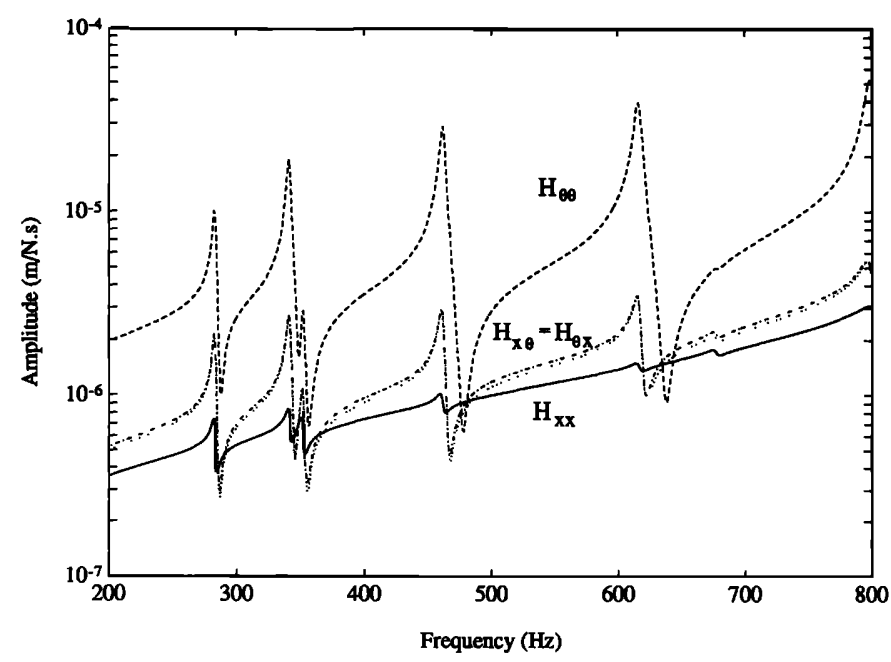

FIG. 4. The admittance characteristics of the simply supported cylinder ( $h_{p}=0.5 \mathrm{~mm}$ ). 


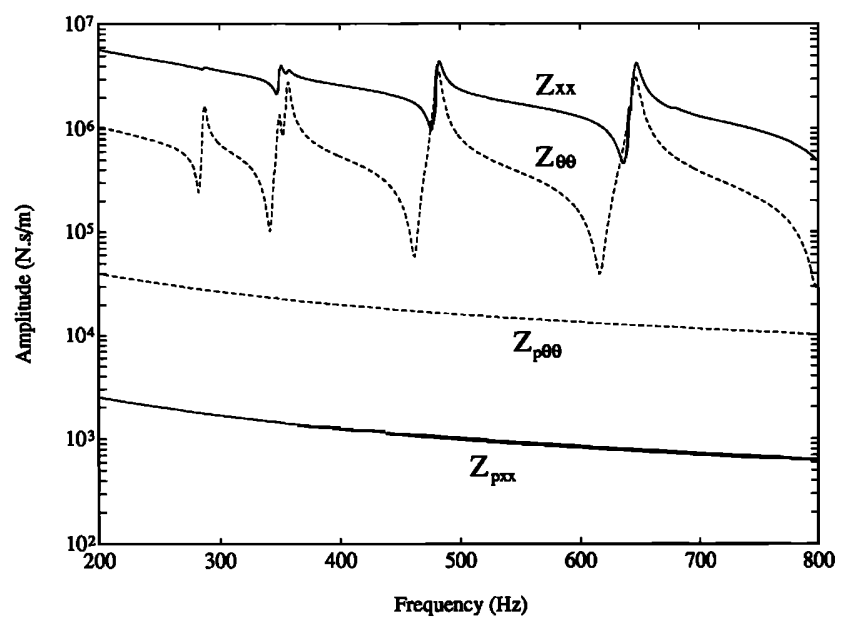

FIG. 5. The direct impedance of the cylinder and the PZT actuator, $h_{p}=0.2$ mm.

several formulas for the actuation of PZT patches on twodimensional structures (Dimitriadis et al., 1989; Wang and Rogers, 1991; Crawley and Lazarus, 1991). One formula developed from the laminated plate theory (Wang and Rogers, 1991) is used here.

Figure 4 shows the variation of the amplitude of the force admittance with frequency. The peaks of the curves correspond to the resonant frequencies of the original cylinder. When the PZT actuator is much thinner than the cylinder ( $h_{p}=0.2 \mathrm{~mm}$ and $h_{p} / h=1 / 10$ ), the input impedance levels of PZT are much lower than those of the cylinder, as shown in Fig. 5. The corresponding amplitude of the dynamic moments is nearly constant over the whole frequency band, as illustrated in Figs. 6 and $7\left(h_{p}=0.2 \mathrm{~mm}\right)$. This result is expected because relatively small PZT patches "planted" in the substrate do not significantly stiffen the cylinder. On the other hand, the host structural dynamics do not yet apply a strong influence on the actuator moment outputs. In this case, the dynamic interaction between the PZT actuator and the cylinder may be ignored. The active moments given by the

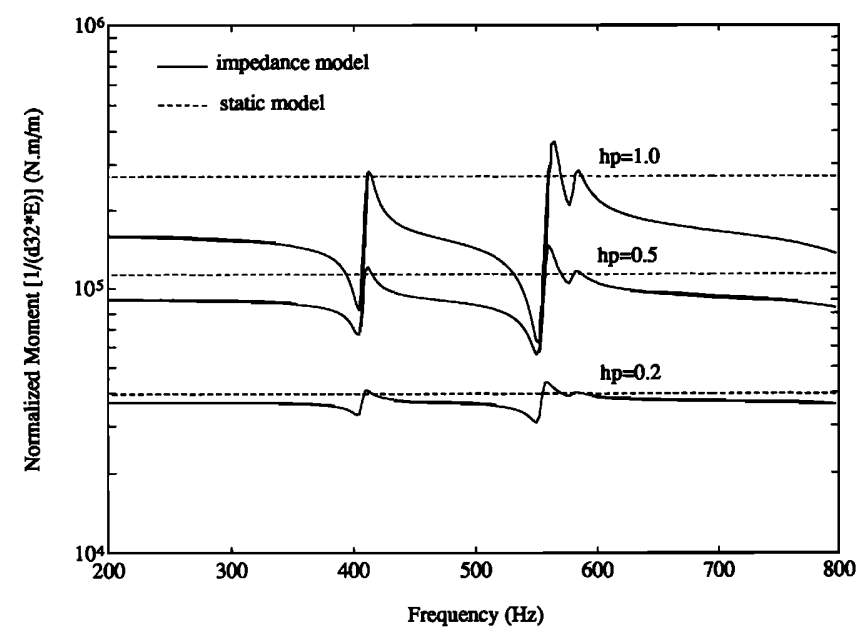

FIG. 6. The moment outputs of the PZT actuator in the circumferential direction.

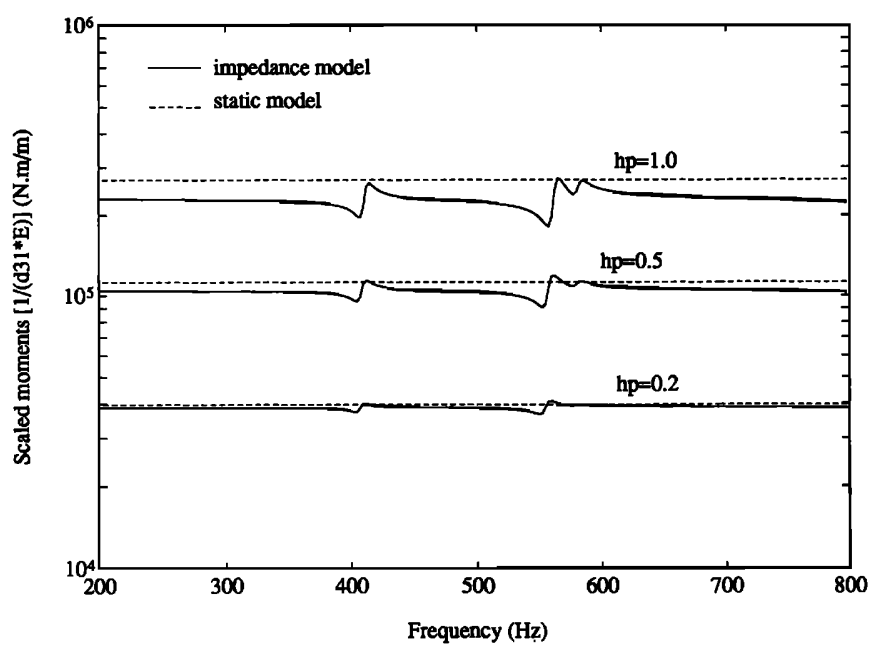

FIG. 7. The moment outputs of the PZT actuator in the axial direction.

static model agrees with that predicted by the impedance model.

With increases in the thickness of the PZT actuator up to $h_{p}=1.0 \mathrm{~mm}\left(h_{p} / h=0.5\right)$, the input impedance of the PZT actuator matches with the structural impedance of the cylinder in the circumferential direction, which is displayed in Fig. 8. The moment outputs of the PZT actuator is greatly strengthened at these matching points. Figure 6 demonstrates that the amplitude of the dynamic moments, $M_{\theta}$, is several times higher than the constant moments predicted by the static approach at some resonance frequencies of the system. The thicker the PZT patches, the greater the increment of the amplitude of the moments. It should be noted that although the axial impedance $Z_{x x}$ in Fig. 8 is not matched by the input impedance $Z_{p \theta \theta}$, the axial moment output $\bar{M}_{x}$ also goes up, as shown in Fig. 7, because of the coupling effect due to the cross impedance and the Poisson's ratio. In addition, Figs. 5 and 8 show that for the cylinder, the mechanical impedance in the axial direction is different from that in the circumferential direction. This implies that the different actuation is

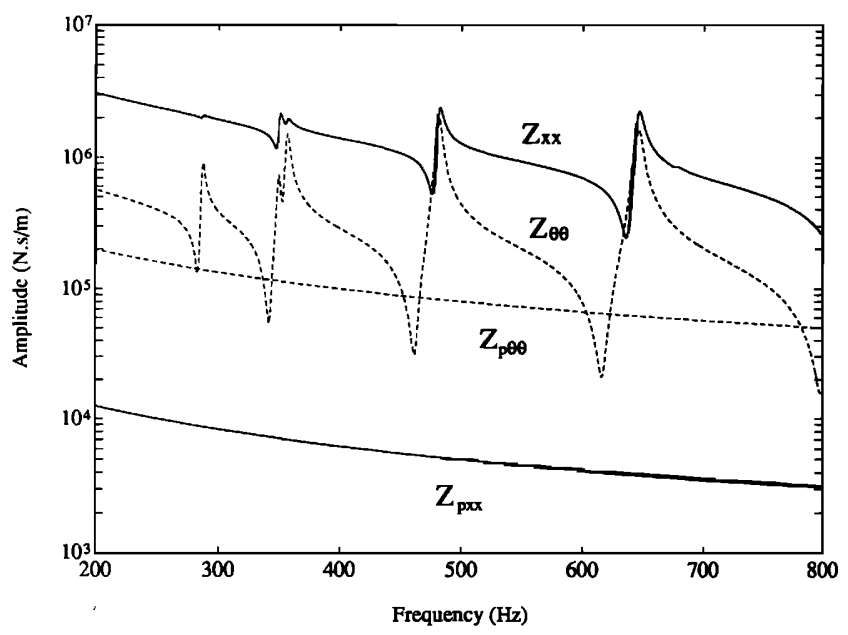

FIG. 8. The direct impedance of the cylinder and the PZT actuator, $h_{p}=1.0$ $\mathrm{mm}$. 

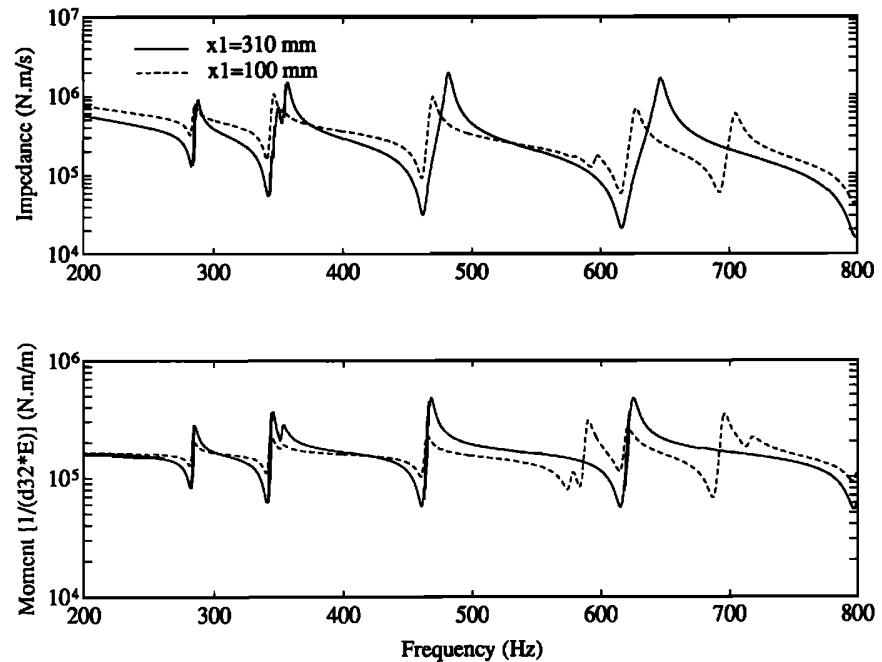

FIG. 9. The effect of the location of the actuator on the circumferential impedance and the moment output $\left(h_{p}=1.0 \mathrm{~mm}\right)$.

generated in the axial direction and in the circumferential direction, i.e., $\bar{M}_{x} \neq \bar{M}_{\theta}$, as illustrated in Figs. 6 and 7 .

The influence of the location of the PZT actuator on the host structure on its dynamic output is examined in Fig. 9. When the location of the excitation points changes, the mechanical impedance of the cylinder varies; accordingly, the moment output of the PZT actuator changes. Since the frequencies corresponding with the peaks of the moments are the resonant frequencies of the entire PZT/shell system, the variation of the peaks due to the location change of the actuator reflects the dynamic performance shifts of the original cylinder. When the PZT actuator is placed on the different locations on the host cylinder, it applies the different stiffening effect on the system. Hence, the location of the PZT actuator is a critical factor affecting the excitation of the actuator on vibrational modes of the system. If the center of the PZT patch is intended to be placed on the nodal line of a certain mode of a host structure, this mode can't be excited. This issue will be further addressed in the following discussion of the dynamic response of the integrated system.

For the convenience of comparing different modeling approaches, a scaled displacement is defined by

$$
w_{\text {normal }}=w / d_{1,(2)} V
$$

so that the effect of the electric parameters of PZT actuators in the modeling can be eliminated. The displacement response of the cylinder is picked up at $x=300 \mathrm{~mm}$ and $\theta=90^{\circ}$. As the thickness of the PZT actuator increases, the natural frequencies of the integrated PZT/cylinder system move up, which is accurately predicted in the impedance model. Figure 10 displays the differences in the displacement responses of the cylinder predicted by the static model and by the impedance model, respectively. The resonant frequencies of the integrated system are shifted to a higher value compared with that of the original cylinder. The corresponding amplitude of the response decreases. It can be explained that thicker PZT patches bonded on the surface of the cylinder stiffen the cylinder, which leads to the variations in the performance of the original structure. The intensity of these

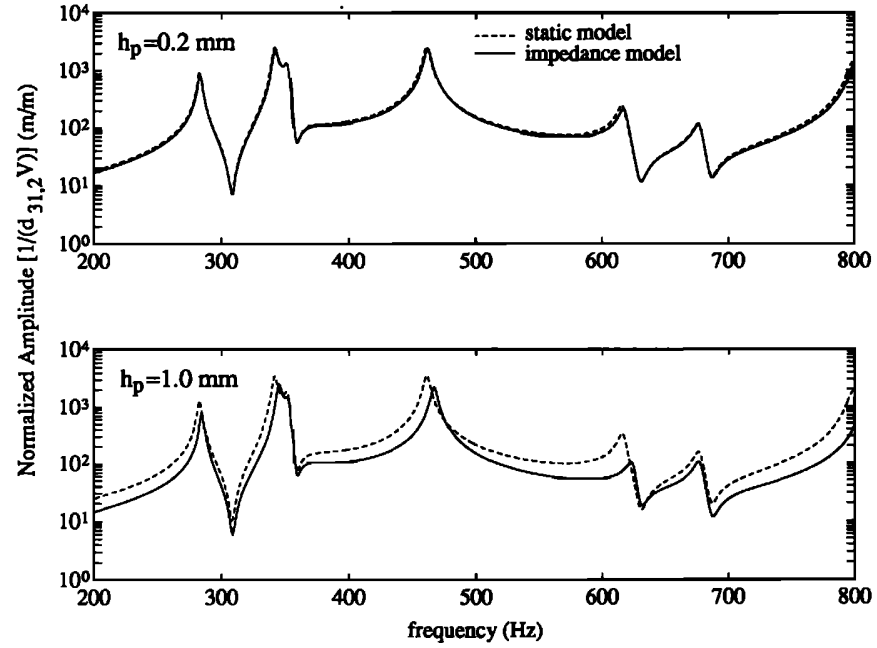

FIG. 10. A comparison of the displacement response of the cylinder predicted by the static model and the impedance model, respectively $(x=310$ $\mathrm{mm}$ and $\theta=90^{\circ}$ ).

changes depends on the extent of the impedance matching between the PZT and the cylinder. When the response happens to be picked up at the nodal line of the 4th mode of the original cylinder $\left(x=310 \mathrm{~mm}\right.$ and $\left.\theta=15^{\circ}\right)$, a significant difference is observed between the different modeling approaches. The static model apparently misses this mode, as shown in Fig. 11. The developed impedance model, however, picks up this mode. In fact, the dynamic performance of the original cylinder has already been shifted by the added PZT actuator and the sensor location $\left(x=310 \mathrm{~mm}\right.$ and $\left.\theta=15^{\circ}\right)$ is not the real nodal line of the 4 th mode of the integrated system. The frequency response function of the entire structure thus reflects this 4 th mode.

\section{SUMMARY}

A theory development using impedance modeling method for the actuation of two-dimensional structures has been performed. The impedance method reveals physics of

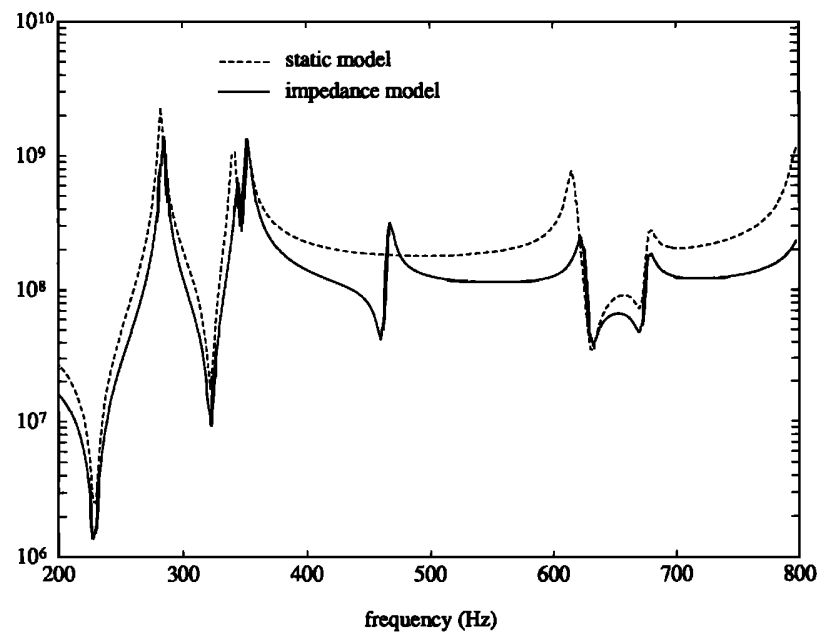

FIG. 11. A comparison of the displacement response of the cylinder predicted by the static model and the impedance model, respectively ( $x=310$ $\mathrm{mm}$ and $\theta=15^{\circ}, h_{p}=1.0 \mathrm{~mm}$ ). 
the dynamic interaction between the actuators and host structures, therefore, it gives more accurate prediction of the output moments of induced strain actuators than the conventional static approach.

The output moments (or forces) of induced strain actuators are strongly related to the input impedance of the actuators and the mechanical impedance of the host structure, and they are frequency dependent.

When the actuator input impedance levels approach or match with the host structural impedance levels, the dynamic performance of the original shell structure is altered because of the stiffening effect of the integrated PZT actuators. The intensity of the stiffening effect varies with the location and the thickness of the PZT actuators.

\section{ACKNOWLEDGMENTS}

The authors gratefully acknowledge the support of the Office of Naval Research, under Grant No. ONR 00014-921170, Dr. K. Ng, Program Manager.

Crawley, E. F. and de Luis, J. (1987). "Use of Piezoelectric Actuators as Elements of Intelligent Structures," AIAA J. 25 (10), 1373-1385.

Crawley, E. F., and Lazarus, K. B. (1991). "Induced Strain Actuation of Isotropic and Anisotropic Plates," AIAA J. 29 (6), 944-951.

Dimitriadis, E. K., Fuller, C. R., and Rogers, C. A. (1989). "Piezoelectric Actuators for Distributed Noise and Vibration Excitation of Thin Plates," ASME Failure Prevent. Reliabil. 16, 223-233.

Hagood, N. W., Chung, W. H., and von Flotow, A. (1990). "Modeling of
Piezoelectric Actuator Dynamics for Active Structural Control," in Proceedings of the 31th SDM (AIAA, Washington, DC), AIAA-90-1097-CP, pp. 2242-2256.

Leissa, W. (1973). Vibration of Shells (NASA, U. S. Government Printing Office, Washington, DC), pp. 31-157.

Lester, H. C. and Lefebvre, S. (1991). "Piezoelectric Actuator Models for Active Sound and Vibration Control of Cylinders," in Proceedings of Recent Advances in Active Control of Sound and Vibration (Technomic, Blacksburg, VA), pp. 3-26.

Liang, C., Sun, F. P., and Rogers, C. A. (1993). "Dynamic Output Characteristics of Piezoceramic Actuators," in Proceedings of Smart Structures and Materials (SPIE, Albuquerque, NM), Vol. 1917, pp. 286-298.

Piezo Systems, Inc. (1987). Piezoelectric Motor/Actuator Kit Manual.

Rossi, A., Liang, C., and Rogers, C. A. (1993). "Coupled ElectricMechanical Analysis of a Piezoceramic Actuator Driven System-An Application to a Circular Ring," in Proceedings of the AIAA/ASME/ASCE/ AHS 34th SDM Conference (AIAA, Washington, DC), La Jolla, CA, 19-21 April, 3618-3624.

Soedel, W. (1981). Vibrations of Shells and Plates (Marcel Dekker, New York), pp. 199-228 and 248-260.

Sonti, V. R., and Jones, J. D. (1991). "Active Vibration Control of Thin Cylindrical Shells Using Piezo-Electric Actuators," in Proceedings of Recent Advances in Active Control of Sound and Vibration (Technomic, Blacksburg, VA), pp. 27-38.

Tzou, H. S. (1989). "Theoretical Development of a Layered Shell with Internal Distributed Controllers," ASME Failure Prevent. Reliabil. 16, 241-249.

Wang, B. T., and Rogers, C. A. (1991). "Modeling of Finite-Length Spatially Distributed Induced Strain Actuators for Laminate Beams and Plates," in Proceedings of the AIAA/ASME/ASCE/AHS 32th SDM Conference (AIAA, Washington, DC), Baltimore, MD, 8-10 April, pp. 15111520. 